\title{
Hilfe durch Gemeindeschwestern, interdisziplinäres Diabetesteam und Essensunterlage
}

\author{
Zum zweiten Mal wurde anlässlich der 6. Herbsttagung der Deutschen Diabetes-Gesellschaft der \\ SilverStar-Förderpreis verliehen. Drei Projekte wurden ausgezeichnet.
}

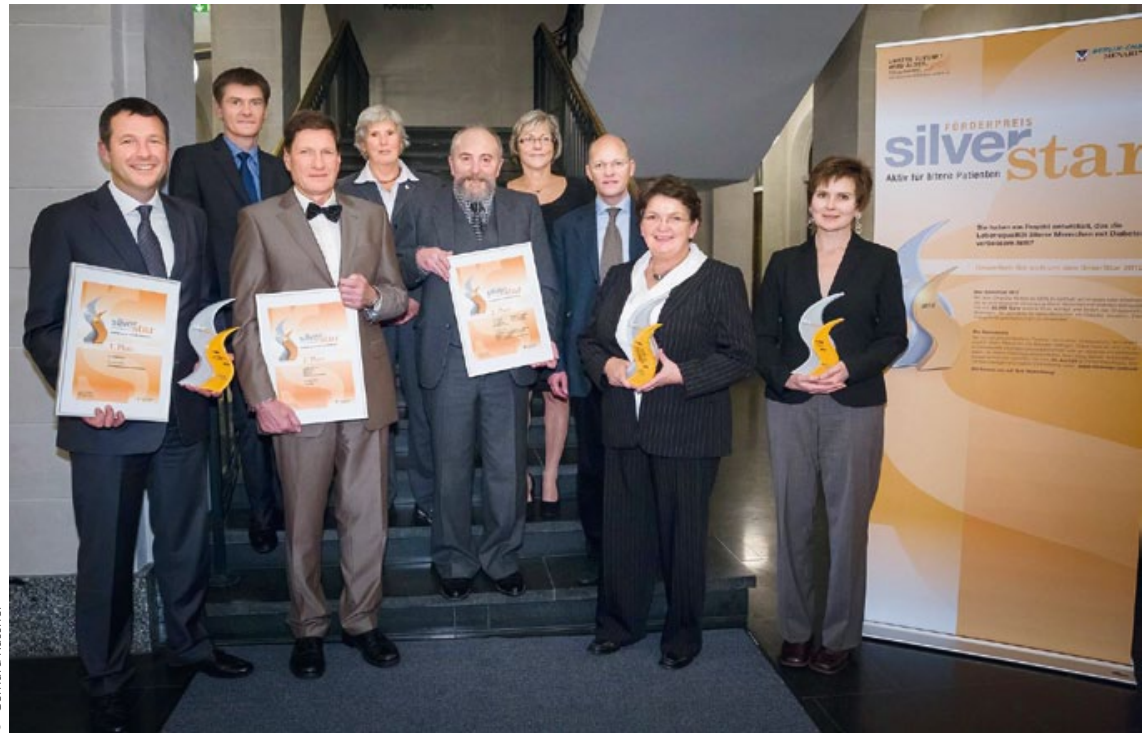

Die Preisträger und der Überbringer bei der Preisverleihung in Berlin, v.l.n.r.: Dr. med. Detlef Kuhn, Torsten Flöttmann (Berlin Chemie), Dr. med. Michael Herr, Dr. Ortrud Hamann, Peter Grau, Hilde Herr, Prof. Dr. med. Cornel Sieber, Irene Feucht.

Der von Berlin Chemie gestiftete Preis mit insgesamt 25000 Euro Preisgeld wird jährlich vergeben. Ziel ist es, Initiativen zu fördern, die ältere Patienten mit Diabetes im Alltag unterstützen. Beworben haben sich über fünfzig Projektgruppen. Die Förderanträge wurden von einer Jury bewertet, der unter anderem der Diabetologe Prof. Rüdiger Petzoldt und der Geriater Prof. Cornel Sieber angehörten.

Der erste Preis (12500 Euro) ging an das Projekt "Gemeindeschwester Muschenheim", das von dem Arzt Dr. Detlef Kuhn in Muschenheim ins Leben gerufen wurde. Die Bürger vom Stadtteil Muschenheim in Lich haben einen Verein gegründet, der über Mitgliedsbeiträge zwei Gemeindeschwestern finanziert. Sie kümmern sich in Kooperation mit den jeweiligen Ärzten zu festgelegten Sprechzeiten um die Betreuung älterer Menschen und speziell Diabetespatienten. $\mathrm{Zu}$ den Aufgaben der Gemeindeschwestern, die selbst Teil der Dorfgemeinschaft sind, gehören Therapiekontrollen und Blutzu- ckermessung, aber auch Schulungen, persönliche Zuwendung und das Lösen von Alltagsproblemen. Praktisch läuft das Projekt so ab, dass die teilnehmenden Hausärzte die Patienten an die Gemeindeschwester vermitteln. „Die Bürger haben aber auch die Möglichkeit, sich direkt bei den Gemeindeschwestern anzumelden, die dann Rücksprache mit dem Hausarzt halten", so Kuhn. Behandelt wird im Gemeindezentrum. Bei Bedarf gibt es auch Hausbesuche. Immer dann, wenn medizinische Probleme auftreten, wird der Arzt hinzugezogen. Derzeit gehören rund 100 alte Menschen zum „Patientenstamm" der Schwestern, viel mehr als anfangs erwartet: „Die Hausärzte werden oft von Patienten aus anderen Dörfern gefragt, warum dieses Projekt nicht auch bei ihnen stattfindet", so Kuhn.

Der zweite Preis (7500 Euro) ging an das Projekt „Pro Edith“ am Marienkrankenhaus in Schwerte. Dort wurde ein fachabteilungsübergreifendes Diabetesteam gebildet. Es begleitet Patienten mit der Nebendiagnose Diabetes während des stationären Aufenthalts und übernimmt koordinierende Funktionen beim Übergang in die ambulante Betreuung.

Ebenfalls mit einem zweiten Preis (5000 Euro) ausgezeichnet wurde eine Initiative des Agaplesion Bethesda Krankenhauses Stuttgart. In dieser Klinik erhalten Diabetespatienten noch während des stationären Aufenthalts eine Essensunterlage, auf der der richtige Umgang mit der Insulintherapie grafisch und in Worten dargestellt ist. Diese Unterlage können die Patienten später mit nach Hause nehmen und haben die wichtigsten Informationen so immer zur Hand.

Weitere Infos und Bewerbung um den Silverstar 2013 unter: www.silverstar-preis.de

Philipp Grätzel von Grätz, Berlin

Quelle: Preisverleihung Silverstar am 16.11.2012 in Berlin, Veranstalter: Berlin Chemie
Gliptin nun auch als Fixkombi und mit Insulin

Mit Komboglyze ${ }^{\circledast}$ ist seit Mitte November eine Fixkombination aus dem DPP-4-Hemmer Saxagliptin $\left(\right.$ Onglyza $\left.{ }^{\oplus}\right)$ und Metformin in Deutschland verfügbar. Parallel dazu erhielt das neue präparat die Zulassung für die Anwendung mit Insulin. Die Fixkombination ist zur Verbesserung der Blutzuckerkontrolle bei Patienten ab 18 Jahren mit Typ-2-Diabetes zugelassen [1]. Es gibt zwei Dosierungen: 2,5 mg Saxa plus 850 mg Met, bzw. 2,5 mg Saxa plus 1000 mg Met. Komboglyze ${ }^{\oplus}$ ist auch mit Insulin als Ergänzung zu Diät und Bewegung angezeigt, wenn Insulin und Metformin allein nicht ausreichen. Bei leichter Niereninsuffizienz ist keine Dosisanpassung nötig. Bei mäßiger bis schwerer ist Komboglyze ${ }^{\oplus}$ kontraindiziert [1].

Quelle: BMS und Astra Zeneca 